\title{
Détermination des caractéristiques géométriques de la croissance d'une bulle de vapeur et des transferts de chaleur associés : influence des incondensables sur le déclenchement d'instabilités convectives
}

\author{
Magali Barthes ${ }^{a}$, Christelle Reynard, Robert Santini et Lounès Tadrist \\ Laboratoire IUSTI, CNRS UMR 6595, DME, 5 rue Enrico Fermi, 13453 Marseille Cedex 13, France
}

Reçu le 25 février 2005, accepté le 21 mars 2005

\begin{abstract}
Résumé - Le présent travail concerne une étude expérimentale de la croissance d'une bulle de vapeur unique dans un bain de FC-72 sous refroidi à pression atmosphérique. La configuration physique encore peu rencontrée dans la littérature se caractérise par des effets gravitationnels stabilisants : la bulle croît sous un élément chauffant (à flux pariétal imposé constant) à partir d'un site de nucléation artificiel indenté en son centre. Une étude de la dynamique de la croissance de la bulle est effectuée à partir d'un traitement d'images permettant d'accéder à l'évolution temporelle des différents paramètres géométriques associés à la bulle, tels que le diamètre maximal, la hauteur, le volume, les contours. Une analyse de certains paramètres permet de montrer notamment l'influence de la puissance de chauffe sur les transferts de masse et de chaleur. Par ailleurs, une observation par ombroscopie des différents modes d'instabilités convectives est présentée. L'influence de la présence d'incondensables sur le déclenchement de l'instabilité est discutée.
\end{abstract}

Mots clés : Ébullition / bulle unique / flux thermiques / instabilités convectives / incondensables

\begin{abstract}
Geometric parameters determination of a single vapor bubble growth and heat transfer associated: non condensable influence on the onset of convective instabilities. We present here an experimental work of a single vapor bubble growth in a subcooled liquid bulk (FC-72) at atmospheric pressure. The vapor bubble grows on a downward facing heating element (at constant heating power) on an artificial nucleation site located in the centre of the heated surface. Bubble dynamics are studied thanks to image proceeding. The temporal evolution of geometric parameters, such as diameter, height, volume and shape, are measured. The analysis of some parameters enables us to determine the influence of the heating power on the heat and mass transfers. Moreover an observation, using a shadowgraphy method, of the different modes of convective instabilities is presented. The non condensable gas influence on the occurrence of the instability is discussed.
\end{abstract}

Key words: Boiling / single bubble / heat flux / convective instabilities / non condensable

\section{Introduction}

Malgré un grand nombre de travaux de recherche fondamentale effectués depuis les années 60 sur le phénomène complexe d'ébullition, de nombreuses questions restent encore ouvertes. Notamment une meilleure compréhension du phénomène permettrait d'expliquer l'origine de l'importance des transferts thermiques générés pour une faible surchauffe à la paroi sous des niveaux différents de gravité. Ceci représente un enjeu

\footnotetext{
a Auteur correspondant :

magali.barthes@polytech.univ-mrs.fr
}

technologique, spécialement dans le domaine spatial où l'énergie thermique à dissiper et la miniaturisation des éléments sont de mises.

Actuellement les études expérimentales effectuées évoluent vers des mesures de plus en plus locales. À cet effet, une première tendance consiste à étudier la croissance de bulles et les transferts de chaleur associés à partir d'une matrice de micro-éléments maintenus généralement à des températures de paroi constantes [1]. La deuxième tendance qui se dégage a trait à des travaux sur des éléments chauffants ne comportant qu'un seul site de nucléation actif [2]. Notre étude s'inscrit dans ce cadre. 


\section{Nomenclature}

\begin{tabular}{|ll|}
\hline$L_{v}$ & Chaleur latente de vaporisation, ${\mathrm{J} . \mathrm{kg}^{-1}}^{-1}$ \\
$P_{\mathrm{c}}$ & Puissance de chauffe, $\mathrm{W}$ \\
$t$ & temps, $\mathrm{s}$ \\
$T_{1}$ & Température du liquide, ${ }^{\circ} \mathrm{C}$ \\
$T_{\text {sat }}$ & Température de saturation, ${ }^{\circ} \mathrm{C}$ \\
$V$ & Volume, $\mathrm{m}^{3}$ \\
$\Delta T_{\text {sub }}$ & Sous refroidissement $\left(\Delta T_{\text {sub }}=T_{\text {sat }}-T_{1}\right),{ }^{\circ} \mathrm{C}$ \\
$\rho_{v}$ & Masse volumique de la vapeur, $\mathrm{kg} \cdot \mathrm{m}^{-3}$ \\
$\Psi$ & Flux, W \\
$\varnothing$ & Diamètre, mm \\
\hline
\end{tabular}

Par ailleurs nous travaillons dans une configuration physique qui reste encore peu testée de nos jours. En effet dans cette expérience, la production de la bulle de vapeur unique a lieu sous un élément chauffant, en son centre, à partir d'un site de nucléation artificiel sous forme de cavité conique. Ce type de configuration a été initié notamment pour l'étude d'un mécanisme particulier pouvant potentiellement contribuer aux transferts de chaleur associés au phénomène d'ébullition, et dont l'origine est indépendante de la gravité : à savoir l'effet Marangoni. Dans les années 90, Straub et al. [3] ont étudié ainsi la convection thermocapillaire avec et sans changement de phase liquide-vapeur sous gravité normale et en microgravité. L'apparition et le rôle de l'effet Marangoni autour d'une bulle d'air représentent une thématique étudiée depuis plusieurs années au laboratoire, et qui est encore d'actualité [4]. En effet, les différentes conclusions avancées sont contradictoires notamment en ce qui concerne l'influence des incondensables lors du changement de phase dans des conditions de sous saturation. C'est pourquoi nous nous intéressons actuellement, avec ou sans dégazage, à la dynamique de croissance d'une bulle de vapeur dans un bain de liquide à sous refroidissement imposé variable.

Nous présenterons tout d'abord l'influence de la puissance de chauffe sur l'évolution temporelle des paramètres géométriques bruts obtenus à partir d'un traitement d'images. Nous discuterons ensuite des transferts de masse déterminés à partir de la variation du volume. Nous terminerons par la visualisation d'instabilités convectives se déclenchant autour de la bulle à partir d'un seuil critique en présence de FC-72 non dégazé et nous discuterons de la nature de ces instabilités.

\section{Dispositif expérimental}

Le dispositif expérimental utilisé est donné en figure 1. L'ébullition sous saturée est réalisée dans une cellule de test entièrement remplie de Fluorinert FC-72 $\left(T_{\text {sat }}=56,6^{\circ} \mathrm{C}, 1 \mathrm{~atm}\right)$. Six hublots (plexiglas ou acier) permettent la visualisation dans différents plans ou supportent l'instrumentation. Le contrôle de la température du bain est assuré par des résistances chauffantes associées à une boucle de régulation (PID, thermocouple). Un peigne de thermocouples, disposés en $\mathrm{T}$ sous la surface de nucléation, permet de déterminer le champ de température dans le bain. Enfin, un élément chauffant, lieu de génération de la bulle, est introduit au sein du liquide. Il est composé d'une résistance chauffante plate (qui assure la surchauffe nécessaire à l'apparition du germe de vapeur) et d'un capteur thermique instrumenté par 7 thermocouples. La bulle de vapeur est créée de manière unique sous cet élément qui est indenté mécaniquement en son centre $(\varnothing=250 \mu \mathrm{m}$, angle au sommet $\left.=45^{\circ}\right)$. La surface de nucléation est située 5,5 mm au-dessus du peigne de thermocouples et est inclinée de $1,8^{\circ}$ afin de permettre le départ de la bulle. Cette configuration originale permet d'obtenir des bulles de tailles contrôlées avec des vitesses de croissance plus lentes (jusqu'à 45 min suivant les conditions opératoires). Ceci diffère du comportement d'une bulle créée au-dessus de l'élément chauffant (temps de croissance très inférieurs à la seconde, [5]). Les pertes par convection et conduction (le long du support de l'élément chauffant) ont été estimées expérimentalement, à partir d'un bilan thermique, à $35 \%$ de la puissance totale, dans les cas les plus défavorables.

La cellule est intégrée dans une boucle fermée totalement étanche comportant un vase d'expansion. Il nous est ainsi possible de travailler à des températures de liquide inférieures à la température de saturation (volume total variable), à pression atmosphérique et en minimisant le taux d'incondensables. Une acquisition vidéo latérale est réalisée grâce à une caméra couplée à un zoom macro, et reliée à une carte d'acquisition. Le type d'éclairage utilisé varie selon le type d'observation souhaitée : éclairage parallèle (ombroscopie) afin de mettre en évidence les gradients d'indice, et ainsi les instabilités convectives; ou bien éclairage diffus afin de déterminer les caractéristiques géométriques de la bulle. En effet, avec un éclairage parallèle, le gradient thermique étant important au voisinage de la paroi, il s'en suit une déformation optique conséquente et le faisceau parallèle est fortement dévié verticalement. Ainsi, c'est dans le but de minimiser les problèmes optiques liés au gradient d'indice dans le fluide (mirages) qu'un éclairage diffus (avec une ouverture de diaphragme minimale) a été utilisé. L'ensemble du dispositif expérimental est monté dans un bâti métallique et le banc d'essai, supportant la cellule test, la caméra et la source lumineuse, est inclinable (angle connu précisément et maîtrisé). Lorsque nous souhaitons travailler en s'affranchissant au mieux des incondensables dans le liquide, ce dernier est porté à ébullition durant plusieurs jours. Nous travaillons dans des conditions de sous refroidissement $\left(\Delta T_{\text {sub }}=T_{\text {sat }}-T_{1}\right)$ et de puissance de chauffe imposées. La température du bain mesurée par le « peigne » de thermocouples est relativement homogène [6] (moins de $0,5{ }^{\circ} \mathrm{C}$ d'écart) dans une zone proche $(4 \mathrm{~cm}$ horizontalement, sur 1 à $3 \mathrm{~cm}$ verticalement) de la surface de nucléation. Le niveau de sous refroidissement annoncé ici a été déterminé à partir de la température moyenne du rang horizontal $\left(T_{1}\right)$ des thermocouples du peigne. 


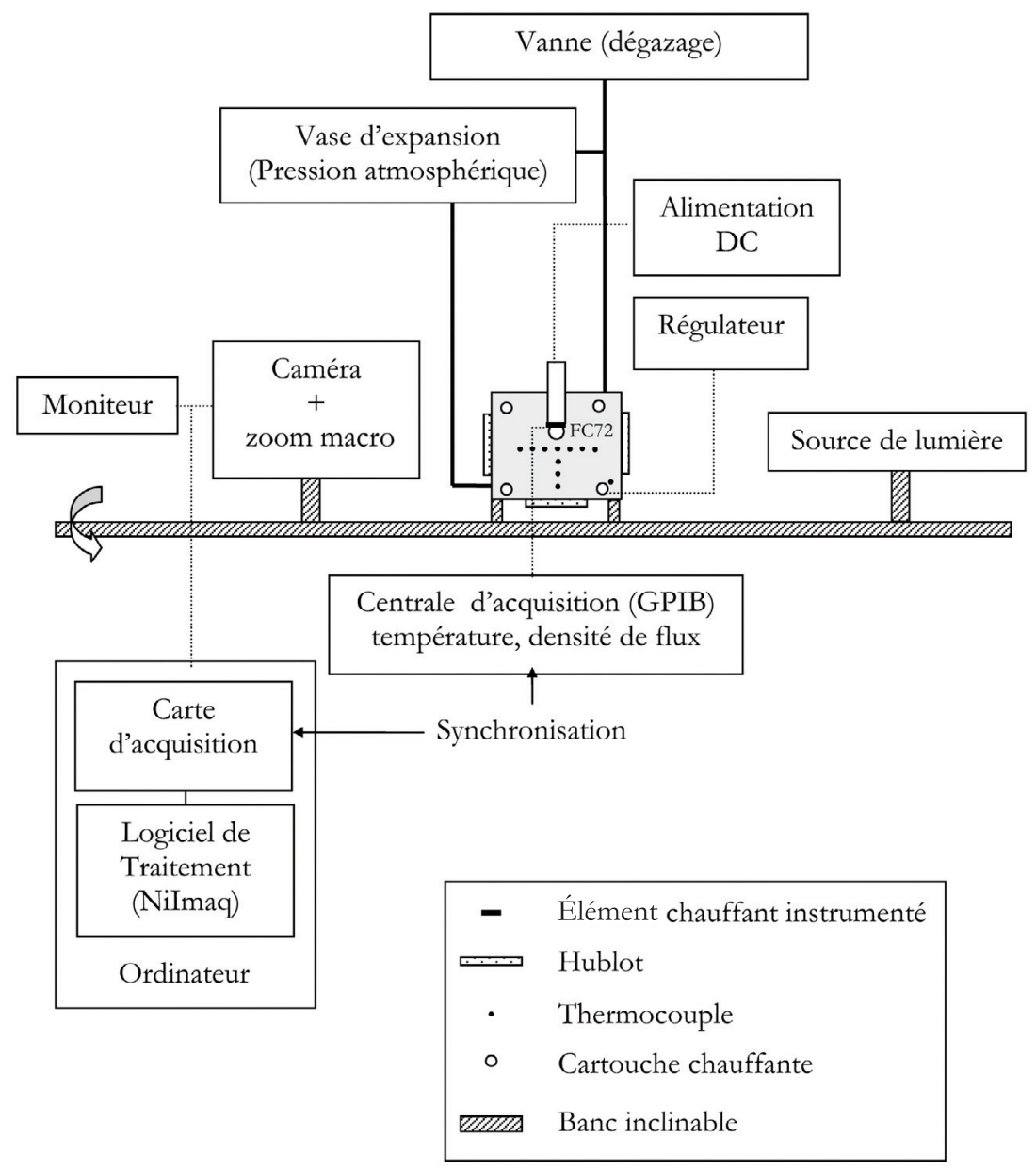

Fig. 1. Schéma du montage expérimental.

\section{Résultats et analyse}

Cette partie concerne la présentation et la discussion de résultats expérimentaux obtenus lors de la croissance d'une bulle de vapeur en faisant varier les paramètres thermiques (sous refroidissement $\Delta T_{\text {sub }}$, puissance de chauffe $P_{\mathrm{c}}$ ) mais aussi certaines des propriétés thermophysiques du fluide sensibles au taux d'incondensables (température de saturation ....). Dans un premier temps, nous nous intéresserons aux paramètres géométriques et à l'influence de la puissance de chauffe sur les transferts thermiques au cours de la croissance de la bulle; puis dans une seconde partie, nous traiterons des instabilités convectives apparaissant dans le liquide au voisinage de l'interface de la bulle, et de l'influence des incondensables sur l'apparition de ces instabilités.

\subsection{Paramètres géométriques et flux associés à la croissance de la bulle de vapeur}

\subsection{1 Évolution temporelle des paramètres géométriques}

L'éclairage diffus est utilisé pour toutes les expérimentations concernant la détermination des paramètres géométriques. Ces derniers (hauteur, diamètre, volume, contour ...) sont mesurés par comptabilisation des pixels grâce à un logiciel développé en Ni-IMAQ ${ }^{\mathrm{TM}}$ couplé à LabVIEW ${ }^{\mathrm{TM}}$. Un exemple des résultats obtenus par traitement d'images est donné en figure 2. À chaque photo de bulle est associée son contour. Sur cette figure 2, le premier contour détectable par traitement d'image (à $t=0,08 \mathrm{~s}$ ) est centré sur le site de nucléation. On constate ensuite que la bulle de vapeur n'est plus centrée sur le site de nucléation : ceci est lié au fait que la surface de nucléation est inclinée de $1,8^{\circ}$ (côté droit de l'image orienté vers le haut). Au bout de $3,28 \mathrm{~s}$, la bulle a atteint une taille importante (soit un diamètre maximal de 1,89 $\mathrm{mm}$ et une hauteur de 1,12 $\mathrm{mm}$ ). Le détachement pour ces conditions expérimentales survient à $t=3,64 \mathrm{~s}$ Le logiciel nous fournit aussi la hauteur, le diamètre etc. (qui peuvent être déterminés aussi à partir du contour); quant au volume, il est calculé en faisant l'hypothèse que la bulle est axisymétrique.

3.1.2 Influence de la puissance de chauffe sur le transfert de masse

Un paramètre thermique important est la puissance de chauffe imposée. Celle-ci a en effet une incidence 


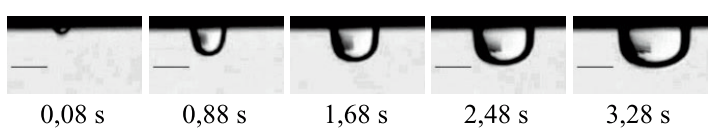

Coordonnées en mm

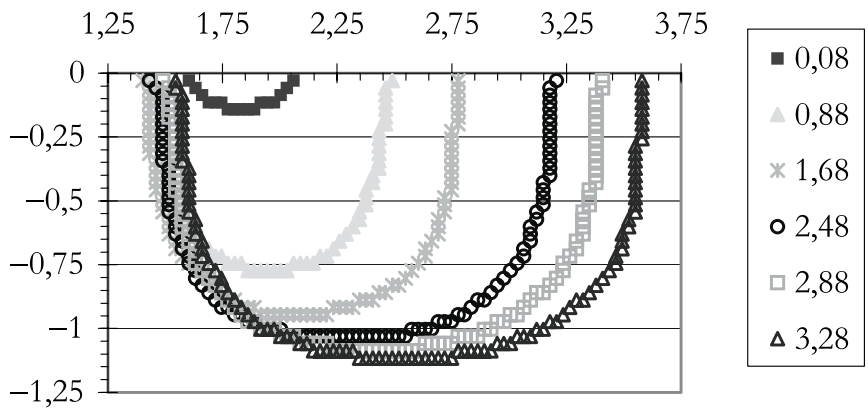

Fig. 2. Photos et contours de la bulle de vapeur à différents instants de sa croissance, $\Delta T_{\mathrm{sub}}=8^{\circ} \mathrm{C}, P_{\mathrm{c}}=655 \mathrm{~mW}$.

directe sur l'évolution temporelle des différents paramètres géométriques. Nous avons mené plusieurs séries d'expérimentations à un sous refroidissement donné $\left(48^{\circ} \mathrm{C}\right)$ et diverses puissances de chauffe $(555,655,755$ et $855 \mathrm{~mW}$ ). Pour ces différentes expériences, l'exploitation de l'évolution temporelle du volume, obtenue grâce au logiciel de traitement d'images, a permis de calculer les flux de chaleur nécessaires au changement de phase liquide-vapeur pour chaque bulle (Éq. (1)) en faisant l'hypothèse que la température de la vapeur est égale à la température de saturation à la pression de travail (pression atmosphérique)

$$
\Psi=L_{v} \rho_{v} \frac{\mathrm{d} V}{\mathrm{~d} t}
$$

Le flux instantané est donné en figure 3a, et le flux total est donné en figure 3b. On constate que le flux total augmente avec la puissance de chauffe : ceci vient du fait qu'il s'agit d'un flux moyenné sur le temps de croissance de la bulle. En effet, la période d'émission de bulle diminue lorsque la puissance de chauffe augmente, de même que le temps de croissance, entraînant par là même une augmentation du flux de changement de phase. Par ailleurs, on remarque que le flux total n'est pas linéaire en fonction de la puissance de chauffe. La fraction d'énergie nécessaire au changement de phase est plus importante pour des puissances de chauffe plus élevées. Pour les flux instantanés on constate que, quelle que soit la puissance de chauffe, l'allure de la courbe est la même. On remarque aussi que le flux instantané croît plus rapidement lorsque la puissance de chauffe est plus importante, et la bulle de vapeur croît plus vite (et donc sa variation temporelle de volume est plus importante).

\subsection{Instabilités convectives : influence de la présence d'incondensables}

Une des difficultés majeures de notre expérimentation est de réussir à s'affranchir de la présence d'incondensables dans notre fluide de test. En effet, lorsque le fluide

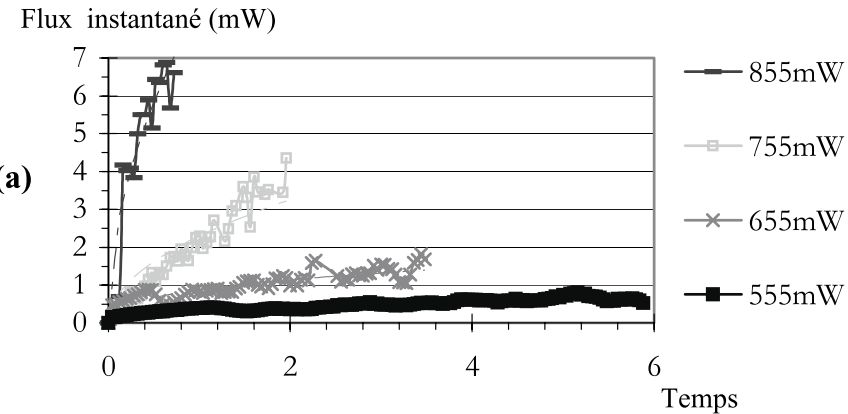

Flux total $(\mathrm{mW})$

(b)

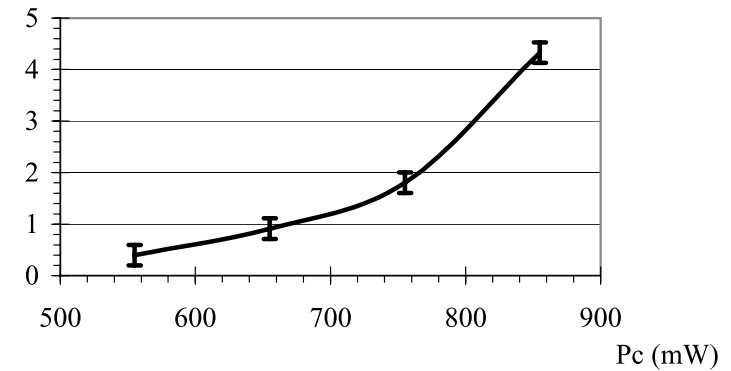

Fig. 3. Flux de changement de phase de la bulle de vapeur pour diverses puissances de chauffe et un même sous refroidissement $\left(8^{\circ} \mathrm{C}\right)$ : (a) instantanés, (b) totaux.

n'est pas du tout dégazé, pour un sous refroidissement donné $\left(\Delta T_{\text {sub }}\right)$ et à partir d'un rayon donné, des instabilités convectives apparaissent. Afin de mettre en évidence ces instabilités, une méthode de diagnostic optique par ombroscopie (mise en évidence du gradient d'indice, et donc de température, dans un fluide par défaut de mise au point) a été utilisée.

\subsubsection{Différents modes de l'instabilité convective}

Des expérimentations antérieures portant sur l'étude de la convection thermocapillaire (effet Marangoni) ont été menées dans notre laboratoire. Il s'agissait d'étudier le déclenchement de ces instabilités autour d'une bulle d'air injectée dans de l'huile de silicone dans un gradient de température imposé. Différents modes avaient été mis en évidence [7] : tout d'abord un mode « symétrique » correspondant à un rouleau primaire déformé périodiquement en un nombre pair d'extremums, puis un mode « pendulaire $»$ (nombre impair d'extremums) et enfin un mode « complexe ».

Dans le cas de la bulle de vapeur, lorsque notre liquide test n'était pas dégazé (c'est-à-dire $T_{\text {sat }}<56,6{ }^{\circ} \mathrm{C}$ ), ces mêmes modes ont été observés avec un mode complexe qui tend rapidement vers un mode chaotique pour lequel les mouvements dans le fluide deviennent violents (phénomènes d'éjection de liquide par «bouffées »). Un exemple de ces différents modes est donné en figure 4, pour un sous refroidissement de $7{ }^{\circ} \mathrm{C}$ et une puissance de chauffe imposée de 1,45 W. Le déclenchement de chacune de ces instabilités se fait pour un rayon de bulle critique donné. Ce dernier est fonction du niveau de sous 

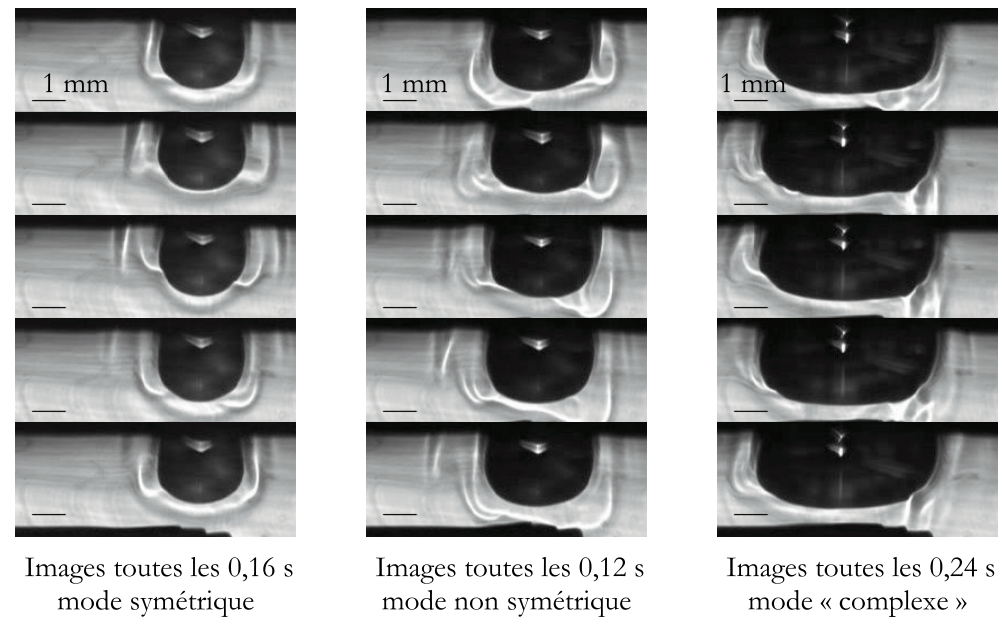

Fig. 4. Différents modes lors de la croissance de la bulle de vapeur, $\Delta T_{\mathrm{sub}}=7^{\circ} \mathrm{C}, P_{\mathrm{c}}=1450 \mathrm{~mW}$.

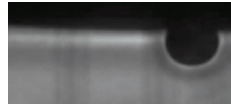

$t=10 \mathrm{~s}$

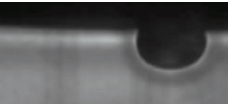

$t=20 \mathrm{~s}$

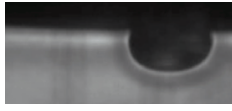

$t=30 \mathrm{~s}$

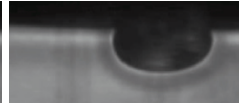

$t=40 \mathrm{~s}$

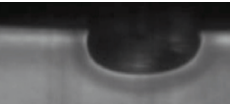

$t=50 \mathrm{~s}$

Fig. 5. Photos en ombroscopie de la croissance de la bulle de vapeur dans un liquide dégazé : aucun mouvement convectif observable, $\Delta T_{\text {sub }}=8{ }^{\circ} \mathrm{C}, P_{\mathrm{c}}=2570 \mathrm{~mW}$.

refroidissement du fluide : ainsi, pour le mode symétrique, le rayon critique de déclenchement augmente lorsque le sous refroidissement diminue. Ce résultat concorde avec celui obtenu autour d'une bulle d'air injectée dans du FC-72 [7]. Ceci nous laisse supposer que ces instabilités convectives sont probablement induites par les effets Marangoni. Selon l'hypothèse émise par Straub et al. [3], pour un liquide sous refroidi, l'origine de ce phénomène serait due à la présence d'incondensables et à des phénomènes de condensation qui engendrent un gradient le long de l'interface de la bulle. Les résultats ont été obtenus ici avec un fluide non dégazé. Afin de déterminer l'influence des incondensables sur l'existence des instabilités convectives, nous avons mené de nouvelles expériences avec un liquide dégazé.

\subsubsection{Influence des incondensables}

Des expériences avec un liquide dégazé au mieux dans les mêmes conditions opératoires qu'avec le liquide non dégazé (sous refroidissement, puissance de chauffe, inclinaison de la surface, pression du système) ont été réalisées. Actuellement, notre montage expérimental ne nous permet pas de mesurer le taux d'incondensables dans le liquide. Afin de s'affranchir au mieux des incondensables, le FC-72 a été porté à ébullition pendant plusieurs jours; lorsque la température de saturation à pression atmosphérique $\left(56,6{ }^{\circ} \mathrm{C}\right)$ a été atteinte dans le liquide, nous avons émis l'hypothèse que le taux d'incondensable dans le liquide était alors négligeable (quasi nul). Nous avons trouvé que, dans ces conditions, les phénomènes d'instabilités convectives n'étaient plus observables. En effet, sur la figure 5, on observe un « halo » stable autour de la bulle de vapeur (résultant du fort gradient thermique) mais aucun mouvement convectif dans le liquide. Ce résultat est en contradiction avec ce que Henry et al. [1] avaient obtenu : en effet, ils pensaient que ces instabilités convectives étaient de type thermocapillaire et qu'elles ne seraient pas dues aux incondensables, mais plutôt à la nature du FC-72 (mélange de plusieurs composants). Selon eux, les incondensables auraient tendance à empêcher le déclenchement de ces instabilités. Une étude en fonction du taux d'incondensables permettrait de déterminer la variation du seuil de déclenchement et ainsi de conclure sur l'influence des incondensables.

\section{Conclusion}

La croissance d'une bulle unique a été observée à partir de deux méthodes optiques. La première associée à un traitement d'images nous a permis de déterminer l'évolution des paramètres géométriques. Nous avons ainsi montré la variation du contour de la bulle en différents instants de sa croissance. Nous obtenons des tailles de bulle conséquentes avec un temps de croissance supérieur à celui obtenu dans le cas d'une bulle créée au-dessus d'un élément chauffant. L'analyse du volume nous a permis de remonter aux flux instantané et total pour différentes puissances de chauffe. Nous avons montré que l'augmentation de ce paramètre thermique entraîne une augmentation du flux total. Avec la seconde méthode optique, la présence d'instabilités convectives a été mise en évidence pour certaines conditions expérimentales. Ainsi nous avons montré que ces instabilités disparaissaient lorsque le fluide était fortement dégazé. Par analogie avec des travaux antérieurs autour de bulle d'air, nous avons 
supposé que ces instabilités sont de type thermocapillaire. Afin de vérifier cette hypothèse, nous étudierons le champ de vitesse autour de la bulle par PIV en faisant varier le taux d'incondensable. Par ailleurs la contribution de cet effet aux transferts de chaleur sera étudiée.

Remerciements. Les auteurs remercient l'ESA et le CNES pour le support financier apporté dans le cadre du contrat CIMEX (Convection and Interfacial Mass Exchange).

\section{Références}

[1] C.D. Henry, J. Kim, B. Chamberlain, Heater size and heater aspect ratio effects on subcooled pool boiling heat transfer in low-g, Proceedings of 3rd International Symposium on Two-Phase Flow Modelling and Experimentation, Pise, Italie, 2004
[2] G. Duhar, C. Colin, Vapor bubble growth and detachment in convective nucleate boiling, Proceedings of 5 th International Conference on Multiphase Flow, ICMF'04, Yokohama, Japon, 2004

[3] J. Straub, Boiling heat transfer and bubble dynamics in microgravity, Advances in Heat Transfer 35 (2001) 57-171

[4] C. Reynard, R. Santini, L. Tadrist, Experimental study of the gravity influence on the periodic thermocapillary convection around a bubble, Experiments in Fluids 31(4) (2001) 440-446

[5] N. Ginet, Analyse des mécanismes contrôlant la croissance et l'ascension d'une bulle isolée en ébullition nucléée, Thèse, INSA, 1999

[6] M. Barthès, C. Reynard, R. Santini, L. Tadrist, Experimental Study of Heat Transfer Induced by a Single Vapor Bubble Growth, Influence of Liquid Subcooling, Proceeding of Space Technology, and Applications International Forum, Albuquerque, États-Unis, 2004

[7] C. Reynard, M. Barthès, R. Santini, L. Tadrist, Experimental study of the onset of the 3rd oscillatory thermocapillary convection around a single air or vapor bubble, Influence on heat transfer, Experimental Thermal and Fluid Science (accepté) 